University of Nebraska - Lincoln

DigitalCommons@University of Nebraska - Lincoln

2008

Small Angle Neutron Scattering Study of Conformation of

Oligo(ethylene glycol)-Grafted Polystyrene in Dilute Solutions: Effect of the Backbone Length

Gang Cheng

Yuri B. Melnichenko

George D. Wignall

Fengjun Hua

Kunlun Hong

See next page for additional authors

Follow this and additional works at: https://digitalcommons.unl.edu/usdoepub

Part of the Bioresource and Agricultural Engineering Commons

Cheng, Gang; Melnichenko, Yuri B.; Wignall, George D.; Hua, Fengjun; Hong, Kunlun; and Mays, Jimmy W., "Small Angle Neutron Scattering Study of Conformation of Oligo(ethylene glycol)-Grafted Polystyrene in Dilute Solutions: Effect of the Backbone Length" (2008). US Department of Energy Publications. 73.

https://digitalcommons.unl.edu/usdoepub/73

This Article is brought to you for free and open access by the U.S. Department of Energy at DigitalCommons@University of Nebraska - Lincoln. It has been accepted for inclusion in US Department of Energy Publications by an authorized administrator of DigitalCommons@University of Nebraska - Lincoln. 


\section{Authors}

Gang Cheng, Yuri B. Melnichenko, George D. Wignall, Fengjun Hua, Kunlun Hong, and Jimmy W. Mays 


\title{
Small Angle Neutron Scattering Study of Conformation of Oligo(ethylene glycol)-Grafted Polystyrene in Dilute Solutions: Effect of the Backbone Length
}

\author{
Gang Cheng, ${ }^{*}, \dagger$ Yuri B. Melnichenko, ${ }^{\dagger}$ George D. Wignall, ${ }^{\dagger}$ Fengjun Hua, ${ }^{\dagger}$ \\ Kunlun Hong, ${ }^{* * *}$ and Jimmy W. Mays ${ }^{*}$
}

Neutron Scattering Science Division and Center for Nanophase Materials Sciences and Chemical Science Division, Oak Ridge National Laboratory, Oak Ridge, Tennessee 37831

Received June 18, 2008; Revised Manuscript Received October 16, 2008

\begin{abstract}
The conformation and clusterization of comblike polymers of polystyrene densely grafted with oligo(ethylene glycol) (OEG) side chains in $1.0 \mathrm{wt} \%$ solutions of $\mathrm{D}_{2} \mathrm{O}$, toluene- $d_{8}$, and methanol- $d_{4}$ was investigated as a function of the degree of polymerization (DP) of the backbone by small angle neutron scattering (SANS). Each side chain had four EG repeat units, and the DP of the polystyrene backbone varied from 8 to 85 . The global conformation of the polymers in toluene and methanol was shown to assume ellipsoidal, rigid cylindrical, or wormlike morphologies with increasing DP of the polystyrene backbone. At the same time, in $\mathrm{D}_{2} \mathrm{O}$, the polymer conformation was described by the form factor of rigid cylinders. The second viral coefficient $A_{2}$ was measured for the polymer with a DP of 85 in all three solvents, and the solvent quality of toluene, methanol, and $\mathrm{D}_{2} \mathrm{O}$ was identified to be good, marginal, and poor, respectively, for this polymer. Because of a poor solvent quality, the PS backbone ( $D P=85)$ is partially collapsed in $\mathrm{D}_{2} \mathrm{O}$, whereas it is moderately expanded in toluene and methanol. Polymers with a DP of 8 were found to form clusters in all three solvents, with the characteristic size between 100 and $200 \AA$ and a fractal dimension of 2 . With the increase in the DP, the clusters diminished in $\mathrm{D}_{2} \mathrm{O}$ and completely disappeared in toluene and methanol. This observation suggests that the clusterization of these short side-chain polymers is caused by end-group and hydrogen bonding interactions between different chains.
\end{abstract}

\section{Introduction}

Comblike polymers have received a great deal of attention because of their unique architecture and versatile conformations. ${ }^{1-10}$ If side chains are long enough, then the backbone becomes highly extended because of the topological stiffness, ${ }^{4}$ and the global conformation corresponds to that of a wormlike chain. Theoretical and computer simulation studies have focused on the wormlike chain regime where scaling relations between the persistence length, the length of the side chains, and the number of the side chains per backbone repeat unit are predicted; however, many of the theoretical predictions cannot be verified because of the limited amount of experimental data and availability of proper comblike polymers. ${ }^{3,5,10 \mathrm{~d}}$ Experimental investigations by SANS and light scattering (LS) have mainly concentrated on hydrophobic comblike polymers where the interactions of backbone and side chains with solvents are not significantly different. The global conformation of polystyrenegrafted polynorbornene comblike polymers has been described as compact spheres or cylinders depending on the DP of the backbone. ${ }^{9}$ Combined SANS and computer simulation studies of $\operatorname{poly}(n$-butyl acrylate) grafted poly(alkyl methacrylate) demonstrate that in a good solvent, a side-chain conformation is close to that of the random coil, the backbone is stretched; and the persistence length increases with the side-chain length until it reaches a limit. ${ }^{3}$ Recent SANS investigations on the conformation of polystyrene-grafted polymethacrylate show a flexible coil to stiff cylinder transition as the length of the side chains increases. ${ }^{5}$

OEG-grafted polymers are promising materials for different applications owing to the special properties of EG in addition to the comblike architecture, which allows independent variation

\footnotetext{
*Corresponding authors. E-mail: chengg@ornl.gov and hongkq@ornl.gov.

${ }^{\dagger}$ Neutron Scattering Science Division.

Center for Nanophase Materials Sciences and Chemical Science Division.
}

of the length and composition of the backbone and side chains to achieve desired structures and functions. A main research activity involves optimization of the properties of OEG-grafted polymers as polymer electrolytes where the OEG side chains are good ionic conductors. ${ }^{11,12}$ Another major thrust has been in the biomedical applications in view of nontoxic, nonimmunogenic properties of EG. It is now widely accepted that the OEG-grafted polymers with hydrophobic backbones have many advantages over poly ( $N$-isopropylacrylamide) (PNIPAM), a well studied model system in biomedical applications. ${ }^{13-15}$ By varying the number of EG repeat unit in the side chain, the cloud point temperature (CPT) of their aqueous solutions can be controlled within the physiological range. ${ }^{15}$

Information on the conformation properties of OEG-grafted polymers at interfaces ${ }^{14,16}$ and in aqueous solutions under different conditions ${ }^{13,17-19}$ is required before their applications can be exploited. SANS and LS are complementary techniques, and both are important in revealing the conformation of the polymers in solutions on the length scale on the order of 10-1000 $\AA$ (SANS) and 100-10 $000 \AA$ (LS), respectively. The contrast between the polymers and the solvent is defined by different physical parameters: the neutron scattering length density (SLD) for SANS and the refractive index for LS. SANS has been largely used for the investigation of the conformation of the polymers in solution because of the appropriate length scales involved as well as the significant contrast between hydrogenated polymers and deuterium-substituted solvents. We previously investigated the conformation of the PS densely grafted with EG chains at a fixed length of the backbone (DP $=30)$ and side chains $(\mathrm{DP}=4)$ in water using SANS. ${ }^{2}$ It was found that the polymers formed clusters in $\mathrm{D}_{2} \mathrm{O}$, whereas they were molecularly dispersed in toluene; the shape of individual polymers could be described as a short cylinder or an ellipsoid.

The major aim of the present work was to study the variation of the polymer conformation as a function of the DP of the backbone (DP $=8,40,47,58,85)$ and fixed length of the side 


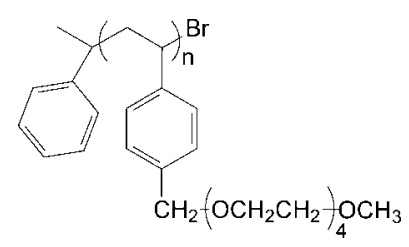

Figure 1. Chemical structure of PTrEGS. $n$ is the DP of the backbone.

EG chains $(\mathrm{DP}=4)$ in various solvents (toluene- $d_{8}$, methanol$d_{4}$, and $\mathrm{D}_{2} \mathrm{O}$ ). One set of experiments was performed at fixed polymer concentration $(1.0 \mathrm{wt} \%)$ and variable DP, and the global conformation of the polymers was assessed in all three solvents by fitting the SANS data to form factors of rigid or semiflexible cylinders (wormlike chains). Another set of experiments was designed to study SANS from polymer solutions with fixed backbone length $(\mathrm{DP}=85)$ as a function of the polymer concentration in the range of 0.2 to $1.0 \mathrm{wt} \%$. These data were used for determining the second virial coefficient in toluene$d_{8}$, methanol- $d_{4}$, and $\mathrm{D}_{2} \mathrm{O}$ to identify the solvent quality. Our studies revealed a strong influence of the end groups on the clusterization of the short-chain polymers in all studied solvents.

\section{Experimental Section}

2.1. Materials. The chemical structure of the homopolymer (abbreviated PTrEGS) is shown in Figure 1. The DP of the polymers is $8,40,47,58$, and 85 , and the polydispersity index is $<1.3$, both determined by gel permeation chromatography. The density of the backbone is assumed to be that of PS, $1.05 \mathrm{~g} / \mathrm{cm}^{3}$, and that of the side chain is assumed to be that of PEG, $1.12 \mathrm{~g} / \mathrm{cm}^{3}$. The polymers were mixed with the solvents and kept for $24 \mathrm{~h}$ at room temperature before the measurements.

2.2. SANS Measurements. SANS measurements were performed at different neutron facilities: the Institute of Materials Research at GKSS, Germany; NCNR at the National Institute of Standard and Technology; and General Purpose SANS instrument (SANS-I) at the HFIR Center for Neutron Scattering at Oak Ridge National Laboratory.

The experiments at NIST were performed on the NG3 30m instrument ${ }^{20 a}$ with a neutron wavelength of $\lambda=6.0 \AA(\Delta \lambda / \lambda \approx$ $0.15)$. Two sample-detector distances were used $(1.5$ and $13.0 \mathrm{~m}$ with a $25 \mathrm{~cm}$ detector offset), which leads to an overall $q$ range of $0.0038 \AA^{-1}<q=4 \pi \lambda^{-1} \sin \theta<0.40 \AA^{-1}$, where $2 \theta$ is the scattering angle. The data were corrected for instrumental background as well as detector efficiency and were put on absolute scale (cross section $I(q)$ in units of $\mathrm{cm}^{-1}$ ) on the basis of the direct beam flux method. Scattering from the solvent was subsequently subtracted in proportion to its volume fraction.

The experiments at ORNL were performed on the SANS-I instrument (http://neutrons.ornl.gov/hfir_instrument_systems/CG-2.shtml) with a neutron wavelength of $\lambda=4.8 \AA(\Delta \lambda / \lambda \approx 0.14)$. Two sample-detector distances were used $(2.8$ and $16.8 \mathrm{~m}$ with a $40 \mathrm{~cm}$ detector offset), which resulted in the overall $q$ range of $0.0045 \AA^{-1}$ $<q=4 \pi \lambda^{-1} \sin \theta<0.45 \AA^{-1}$. The data were corrected for instrumental background as well as detector efficiency and were put on absolute scale (cross section $I(q)$ in units of $\mathrm{cm}^{-1}$ ) by means of precalibrated secondary standard. ${ }^{20 \mathrm{~b}}$ Scattering from the solvent was subsequently subtracted proportionally to its volume fraction.

The experiments at GKSS were performed on the SANS-2 instrument (http://www.gkss.de/central_departments/genf/instruments/ 003124/index_0003124.html.en) with a neutron wavelength of $\lambda=$ $5.8 \AA(\Delta \lambda / \lambda \approx 0.10)$. Two sample-detector distances were used $(0.8$ and $3.8 \mathrm{~m}$ ), which leads to an overall $q$ range of $0.0080 \AA^{-1}<q=$ $4 \pi \lambda^{-1} \sin \theta<0.30 \AA^{-1}$. The data were corrected for instrumental background as well as detector efficiency and put on absolute scale (cross section $I(q)$ in units of $\mathrm{cm}^{-1}$ ) on the basis of a standard sample of a single crystal of vanadium. Scattering from the solvent was subsequently subtracted proportionally to its volume fraction.

The measured neutron scattering intensity in dilute solutions per unit volume is expressed as ${ }^{21}$

$$
I(q)=\frac{c}{K} \frac{\overline{P(q)}}{1+2 c A_{2} M_{\mathrm{w}} \overline{P(q)}}+\mathrm{bkg}
$$

where $c$ is the concentration in $\mathrm{g} / \mathrm{mL}, M_{\mathrm{w}}$ is the weight-average molecular weight, $K=\overline{V_{\mathrm{p}}} \rho / \Delta \rho^{2} \overline{V_{\mathrm{p}}^{2}}, \rho$ is the mass density of a polymer, $\Delta \rho$ is the SLD difference between the polymer and solvent, $V_{\mathrm{p}}$ is the volume of one polymer, and bkg is the incoherent scattering from the polymers. $A_{2}$ is the second virial coefficient that characterizes the average interactions between two polymers in infinitely dilute solutions, $P(q)$ is the form factor, and $P(q=0)$ $=1$. Assuming a Schulz distribution of the particle sizes ${ }^{22}$

$$
f(r)=\frac{r^{z}}{\Gamma(z+1)}\left(\frac{z+1}{<r>}\right)^{z+1} \exp \left[\frac{-r(z+1)}{<r>}\right]
$$

The polydispersity $\sigma$ is given as $\sigma^{2}=1 /(z+1)$.

The averaged form factor is then given as

$$
\overline{P(q)}=\int P(q) V_{\mathrm{p}}^{2} f(r) \mathrm{d} r / \int V_{\mathrm{p}}^{2} f(r) \mathrm{d} r
$$

When $2 c A_{2} M_{\mathrm{w}} \ll 1$, eq 1 is reduced to ${ }^{22}$

$$
I(q)=\frac{\varphi}{V_{\mathrm{p}}}(\Delta \rho)^{2} \overline{V_{\mathrm{p}}^{2}} \overline{P(q)}+\mathrm{bkg}
$$

where $\varphi=c / \rho$ is the volume fraction of the particles.

The $A_{2}$ value was determined via the Zimm plot, $c / I(q=0)$ versus $c$

$$
\frac{c}{I(0)}=K\left(1+2 A_{2} c M_{\mathrm{w}}\right)
$$

The SLD of the PS is $1.4 \times 10^{-6} \AA^{-2}$, and that of the EG is $0.62 \times 10^{-6} \AA^{-2}$. The contrast between $\mathrm{D}_{2} \mathrm{O}\left(6.4 \times 10^{-6} \AA^{-2}\right)$ and each of the component polymers is similar; therefore, SANS detects the global conformation of the polymer.

\section{Results and Discussion}

3.1. Solvent Quality for PTrEGS with a DP of 85. The solution properties of PEG (or poly(ethylene oxide)) in water have been extensively investigated. PEG molecules often form clusters in water, and the origin of this clusterization has not been completely understood. ${ }^{24,25}$ Nevertheless, water has been described as a good solvent for PEG homopolymers. ${ }^{26}$ In the presence of the hydrophobic PS backbone, the distribution of water molecules around PEG side chains is strongly perturbed, and their solubility in water is expected to drop. In fact, it takes more than $10 \mathrm{~h}$ to dissolve $1.0 \mathrm{wt} \%$ PTrEGS in $\mathrm{D}_{2} \mathrm{O}$, and the CPT of this solution $\left(\sim 38^{\circ} \mathrm{C}\right)$ is significantly lower than that of aqueous solutions of PEG (above $100{ }^{\circ} \mathrm{C}^{25}$ ). The CPT of 1.0 wt $\% \mathrm{H}_{2} \mathrm{O}$ solution of DP $=85$ is $\sim 40{ }^{\circ} \mathrm{C}$ because of the better solubility of the polymers in $\mathrm{H}_{2} \mathrm{O}$. It is believed that the solvation of the PTrEGS polymers in water is similar to that of protein globules in which the hydrophobic core is surrounded by a shell of hydrophilic segments. ${ }^{27}$ The PTrEGS polymers are readily dissolved in toluene because of strong interactions between PS backbones and toluene molecules. Methanol molecules have both hydrophilic hydroxyl groups and hydrophobic methyl groups; therefore, the PTrEGS polymers can also be easily dissolved in methanol.

Figure 2 shows the SANS data of $1.0 \mathrm{wt} \%$ polymers with $\mathrm{DP}=85$ in toluene- $d_{8}$, methanol- $d_{4}$, and $\mathrm{D}_{2} \mathrm{O}$. Within the $q$ range investigated, SANS data show no signs of the polymer clusterization in all three solutions. To characterize the solvent quality, the $A_{2}$ values of this polymer were determined in all three solvents by Zimm analysis (eq 4).

The values of $I(0)$ were obtained from SANS data in the concentration range of 0.2 to $1.0 \mathrm{wt} \%$ via Guinier analysis. The maximum polymer concentration $(1.0 \mathrm{wt} \%)$ was much lower than the overlap concentration $(\sim 8.3 \mathrm{wt} \%)$ estimated 


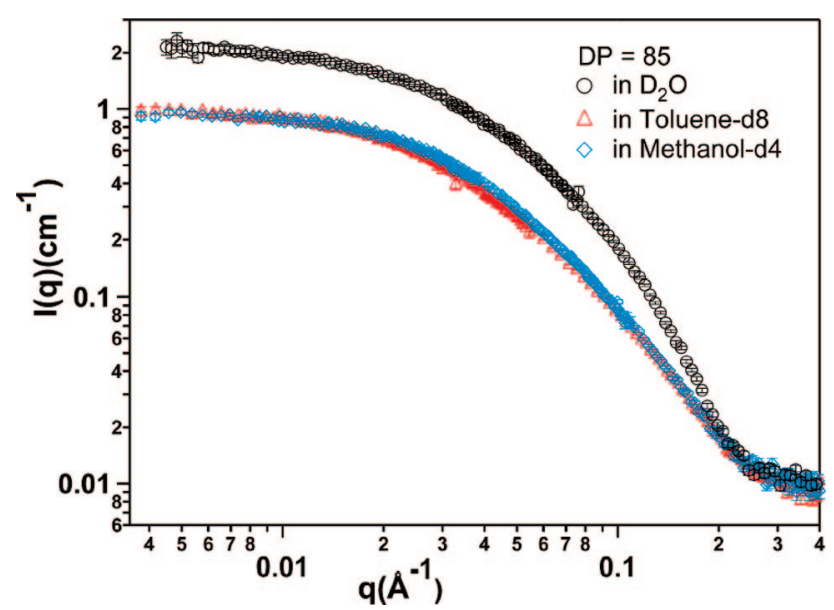

Figure 2. SANS curves of $1.0 \mathrm{wt} \%$ PTrEGS homopolymers with DP $=85$ in $\mathrm{D}_{2} \mathrm{O}$ (data taken at ORNL), toluene- $d_{8}$, and methanol- $d_{4}$ (data taken at NIST).

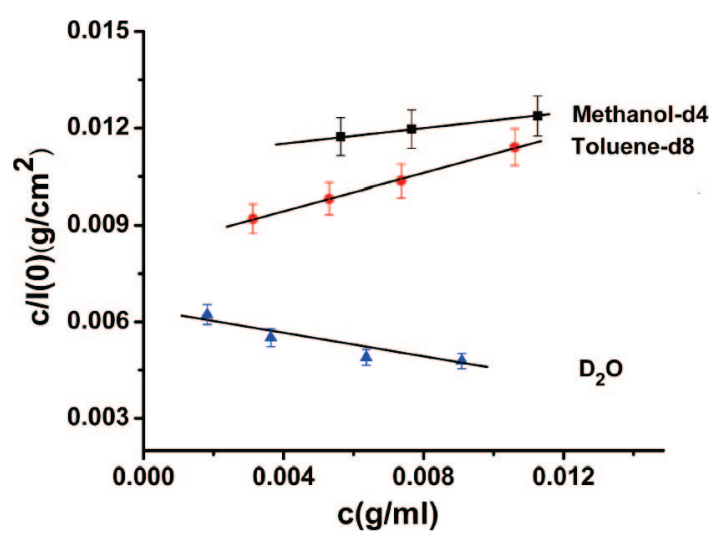

Figure 3. Zimm plot for the polymers with a DP of 85 in three solvents.

via $c^{*}=M_{\mathrm{w}} / N_{\mathrm{A}} V$, where $V=\left(4 \pi R_{\mathrm{g}}{ }^{3}\right) / 3, N_{\mathrm{A}}$ is Avogadro's number, and the radius of gyration $R_{\mathrm{g}}=55 \AA$ was obtained from Guinier analysis of the data of $0.2 \mathrm{wt} \%$ toluene solution.

Zimm plots for PTrEGS solutions in all three solvents are shown in Figure 3. The obtained values of $A_{2}:(5.0 \pm 1.6) \times$ $10^{-4} \mathrm{~mol} \cdot \mathrm{cm}^{3} \cdot \mathrm{g}^{-2}$ (toluene- $\left.d_{8}\right), \quad(1.4 \pm 1.8) \times 10^{-4}$ $\mathrm{mol} \cdot \mathrm{cm}^{3} \cdot \mathrm{g}^{-2}$ (methanol- $\left.d_{4}\right)$, and $-(4.2 \pm 1.1) \times 10^{-4}$ $\mathrm{mol} \cdot \mathrm{cm}^{3} \cdot \mathrm{g}^{-2}\left(\mathrm{D}_{2} \mathrm{O}\right)$ allow us to categorize the solvent quality of toluene- $d_{8}$, methanol- $d_{4}$, and $\mathrm{D}_{2} \mathrm{O}$ for PTrEGS as good, marginal, and poor, respectively. The negative $A_{2}$ value of PTrEGS in $\mathrm{D}_{2} \mathrm{O}$, which is in contrast with positive values of PEG in water, ${ }^{26}$ suggests that the interactions between the polymers in this solvent are attractive, and this is attributed to insolubility of hydrophobic PS backbones in water. Individual homopolymers such as PS are known to collapse into globules in poor solvents, and the globules tend to form clusters because of attractive interactions between polymer segments. ${ }^{27,28}$ The absence of clusters in the aqueous solution of the PTrEGS (DP $=85$ ) is probably due to the availability of the water-soluble EG side chains, which stabilize the solubility of PTrEGS in $\mathrm{D}_{2} \mathrm{O} .{ }^{27}$ However, with the decrease in the DP, as will be discussed later, other factors start to dominate the clusterization. The nearly zero $A_{2}$ suggests that the solubility of the polymers in methanol is between toluene and $\mathrm{D}_{2} \mathrm{O}$. The molecular weight of the polymer, $M_{\mathrm{w}}$, determined from Zimm plots in toluene- $d_{8}$ (27 $800 \mathrm{~g} / \mathrm{mol})$, methanol $-d_{4}(39600 \mathrm{~g} / \mathrm{mol})$, and in $\mathrm{D}_{2} \mathrm{O}(29000$ $\mathrm{g} / \mathrm{mol}$ ) agrees within $20 \%$ with the $M_{\mathrm{w}}$ measured using gel permeation chromatography (34 $800 \mathrm{~g} / \mathrm{mol})$.
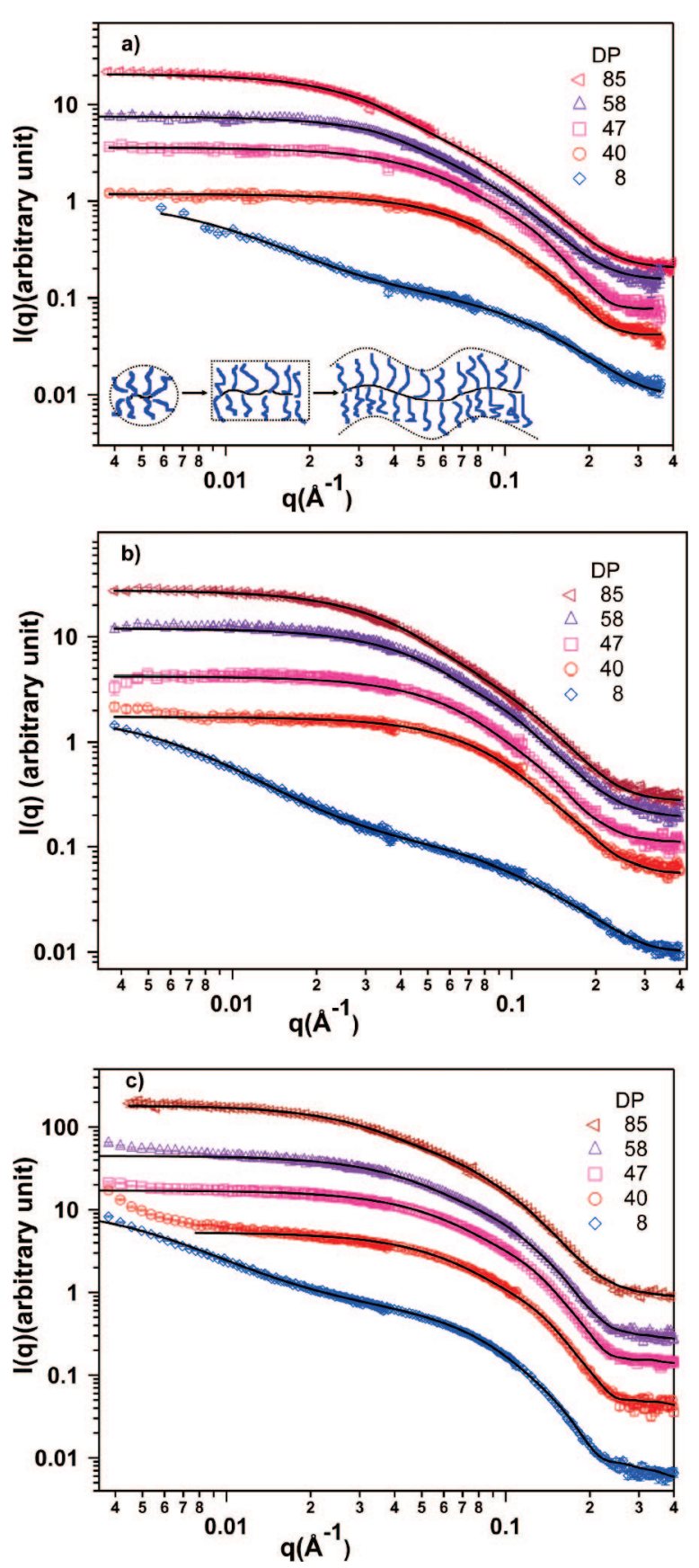

Figure 4. SANS curves of $1.0 \mathrm{wt} \%$ PTrEGS homopolymers with different DPs of the backbone in (a) toluene- $d_{8}$, (b) methanol- $d_{4}$, and (c) $\mathrm{D}_{2} \mathrm{O}$. For the sake of clarity, the data of the toluene solutions were shifted by a factor of $1.2,4,8,14$, and 24 for the polymers of DP $=$ $8,40,47,58$, and 85 , respectively. The data of the methanol solutions were shifted by a factor of $6,12.5,22$, and 30 for the polymers of DP $=40,47,58$, and 85 , respectively. The data of the $\mathrm{D}_{2} \mathrm{O}$ solutions were shifted by a factor of $0.8,5.7,16,32$, and 90 for the polymers of DP $=8,40,47,58$, and 85 , respectively. The inset in a shows the schematic diagram of the shape transition of the polymers with increasing DP. The solid lines are fits to different models, as described in the text.

3.2. Polymer Conformation and Clusterization in $\mathbf{1 . 0}$ wt \% Solutions. To investigate the influence of the DP of the backbone on the conformation of the PTrEGS polymers, we measured SANS from $1.0 \mathrm{wt} \%$ polymer solutions with DP = $8,40,47,58$, and 85 in toluene- $d_{8}$, methanol- $d_{4}$, and $\mathrm{D}_{2} \mathrm{O}$. (See Figure 4.) A striking feature is that the polymers with a DP of 8 formed clusters in all three solvents. The clusters were also detected in $0.1 \mathrm{wt} \%$ toluene solution (data not shown). They were not observed within the experimental window in both toluene- $d_{8}$ and methanol- $d_{4}$ for polymers with a DP of 30 (ref 
2), 40, 47, 58, and 85; in $\mathrm{D}_{2} \mathrm{O}$, the clusterization gradually diminished with the increasing DP. Because the chemical composition of the PTrEGS polymer remains unchanged with DP, it appears that the observed DP-dependent clusterization is induced by interactions between the end groups of the PTrEGS polymers as the relative concentration of the end groups drops with the increase in the DP. In addition, the probability of the end-group interactions drops with increasing DP because of the larger number of conformations available for longer chains. The $A_{2}$ value is related to the interactions between polymers in solution. It is known to be molecular-weight dependent, ${ }^{26,30}$ and the dependence may be altered by the chemical composition of the end groups. ${ }^{24,29}$ As shown in Figure 1, each PTrEGS polymer is terminated by a hydrophobic phenyl group on one end and a bromine atom on the other end. The insoluble hydrophobic end groups tend to avoid contacts with $\mathrm{D}_{2} \mathrm{O}$ molecules and try to interact with the hydrophobic end groups of other molecules. The probability of end-to-end interactions becomes lower with increasing DP because of the mentioned reasons. This is consistent with computer simulations, ${ }^{24}$ which indicate that the attractive interactions increase with the decrease in the molecular weight because of the presence of the hydrophobic end groups in aqueous solutions of methylterminated PEG. In addition to the above-mentioned end-toend interactions, the hydrophobic end groups may also interact with methyl end groups of EG side chains of other polymers, and this type of interaction is shown to be responsible for clusterization of PEG in water. ${ }^{25}$ Finally, the clusterization may be further facilitated by attractive interactions between EG side chains via hydrogen bonding, ${ }^{24}$ where $\mathrm{D}_{2} \mathrm{O}$ molecules act as the physical cross-linkers between side chains that belong to different polymers. Currently, we do not have a convincing explanation of the observed polymer clusterization in good and marginal solvents (toluene and methanol). However, we note that the clusterization in polymer solutions is a common phenomenon and was previously documented in polymer solutions such as PS in cyclohexane around the $\theta$ temperature. ${ }^{31}$

Figure $4 \mathrm{a}, \mathrm{b}$ shows the SANS patterns from the PTrEGS polymers in toluene and in methanol, respectively. The solid lines on the SANS curves are fits to eq 4 . When the backbone length is larger than the persistence length of the polymer, the polymer conformation is better described by the form factor of a semiflexible cylinder. On the contrary, when the backbone length is smaller than the persistence length, the conformation is better described by the form factor of a rigid cylinder. For polymers with DP $=85$ and 58 , a form factor of the semiflexible cylinder $^{32}$ (eq A3 in the Appendix) was used, and the rigid cylinder form factor (eq A1 in the Appendix) was chosen for $\mathrm{DP}=47$ and 40 . We found that the introduction of an additional fitting parameter, polydisperse cross-section radius, was necessary to improve the fits. The reduced $\chi^{2}$ of the fitting was about 2 to 3 .

The SANS data from the polymers with DP of $85,58,47$, and 40 in $\mathrm{D}_{2} \mathrm{O}$ (Figure $4 \mathrm{c}$ ) were consistent with the form factor of the rigid cylinder. The reduced $\chi^{2}$ of the fitting was about 3 to 5. Because of the nonzero values of the second virial coefficient, the values of fitting parameters presented in Table 1 are expected to be concentration dependent. The related corrections to the value of the polymer dimensions depend on the value of the term $2 c A_{2} M_{\mathrm{w}}$; positive $A_{2}$ should lead to an increase in the fitted dimensions of the polymers, whereas negative $A_{2}$ should result in a reduction in the dimensions. For example, when $A_{2}=-(4.2 \pm 1.1) \times 10^{-4} \mathrm{~mol} \cdot \mathrm{cm}^{3} \cdot \mathrm{g}^{-2}$ was included in the fitting (eq 1) of the data from $1.0 \mathrm{wt} \%$ polymers (DP $=85$ ) in $\mathrm{D}_{2} \mathrm{O}$, a $\sim 20 \%$ reduction in the fitted average volume of the cylinders was observed. Unfortunately, systematic measurements of $A_{2}$ for lower-molecular-weight polymers in
Table 1. Fitted Parameters for Polymers in $\mathrm{D}_{2} \mathrm{O}$, Toluene- $d_{8}$, and Methanol- $d_{4}$

Polymers in $\mathrm{D}_{2} \mathrm{O}$

\begin{tabular}{crccc}
\hline DP & \multicolumn{1}{c}{$L(\AA)^{a}$} & $R(\AA)^{b}$ & $\sigma^{c}$ & \\
\hline 85 & $136.2 \pm 0.5$ & $13.3 \pm 0.1$ & $0.27 \pm 0.01$ & \\
58 & $106.2 \pm 0.3$ & $14.1 \pm 0.1$ & $0.22 \pm 0.01$ & \\
47 & $88.0 \pm 0.2$ & $14.6 \pm 0.1$ & $0.15 \pm 0.01$ & \\
40 & $76.2 \pm 0.3$ & $14.9 \pm 0.1$ & $0.09 \pm 0.01$ & $\xi(\AA)^{e}$ \\
\hline & $L(\AA)$ & $R(\AA)$ & $\mathrm{S}(0)^{d}$ & $196.1 \pm 1.6$ \\
\hline 8 & $65.2 \pm 0.3$ & $16.3 \pm 0.1$ & $12.5 \pm 0.2$ & \\
& & Polymers in Toluene- $d_{8}$ & \\
\hline
\end{tabular}

\begin{tabular}{crccc}
\hline $\mathrm{DP}$ & \multicolumn{1}{c}{$L(\AA)$} & $R(\AA)$ & $\sigma$ & $\mathrm{b}(\AA)^{f}$ \\
\hline 85 & $238.5 \pm 0.9$ & $10.5 \pm 0.1$ & $0.30 \pm 0.01$ & $82.7 \pm 1.0$ \\
58 & $133.1 \pm 1.0$ & $10.8 \pm 0.1$ & $0.30 \pm 0.01$ & $82.7 \pm 1.0$ \\
47 & $81.8 \pm 0.5$ & $11.6 \pm 0.2$ & $0.30 \pm 0.01$ & \\
40 & $60.6 \pm 0.4$ & $12.6 \pm 0.1$ & $0.24 \pm 0.01$ & $\xi(\AA)^{e}$ \\
\hline & $R_{\mathrm{a}}(\AA)^{g}$ & $R_{\mathrm{b}}(\AA)^{h}$ & $\mathrm{~S}(0)^{d}$ & $102.9 \pm 2.7$ \\
\hline 8 & $23.9 \pm 0.6$ & $11.8 \pm 0.1$ & $0.7 \pm 0.1$ & \\
& & & \\
& &
\end{tabular}

\begin{tabular}{ccccc}
\hline $\mathrm{DP}$ & $L(\AA)$ & $R(\AA)$ & $\sigma$ & $\mathrm{b}(\AA)^{f}$ \\
\hline 85 & $220.3 \pm 1.7$ & $11.7 \pm 0.1$ & $0.27 \pm 0.01$ & $71.2 \pm 1.3$ \\
58 & $139.9 \pm 0.7$ & $11.6 \pm 0.2$ & $0.24 \pm 0.01$ & $71.2 \pm 1.3$ \\
47 & $76.6 \pm 0.4$ & $12.1 \pm 0.1$ & $0.26 \pm 0.01$ & \\
40 & $60.1 \pm 0.3$ & $12.5 \pm 0.1$ & $0.20 \pm 0.01$ & \\
\hline & $R_{\mathrm{a}}(\AA)^{g}$ & $R_{\mathrm{b}}(\AA)^{h}$ & $\mathrm{~S}(0)^{d}$ & $\xi(\AA)^{e}$ \\
\hline 8 & $31.3 \pm 0.4$ & $10.4 \pm 0.1$ & $1.4 \pm 0.1$ & $151.8 \pm 1.6$
\end{tabular}

${ }^{a}$ Length of a cylinder. ${ }^{b}$ Cross-section radius of a cylinder. ${ }^{c}$ Polydispersity of $R .{ }^{d}$ Related to the susceptibility of the solutions. ${ }^{e}$ Characteristic length of the clusters. ${ }^{f}$ Kuhn length of a semiflexible cylinder. ${ }^{g}$ Rotational semiaxis of an ellipsoid. ${ }^{h}$ Another axis.

$\mathrm{D}_{2} \mathrm{O}$ were not possible because of the strong contribution of scattering from clusters. However, it is expected that the effect of $A_{2}$ on the fitted cylinder volumes of other polymers with lower DP will be within $\sim 20 \%$.

The data presented in Table 1 show that the dimensions of the cylinders or semiflexible cylinders decrease with the DP. For DP $=85$ and 58 , the polymer backbone is in a folded ${ }^{27}$ or crumbled ${ }^{33}$ state in the poor solvent $\mathrm{D}_{2} \mathrm{O}$ in contrast with that in toluene and methanol. This conclusion is not affected by the finite concentration effect because it tends to overestimate the dimensions of the polymers in $\mathrm{D}_{2} \mathrm{O}$ and underestimate the dimensions in toluene and methanol. Further collapse of this polymer in $\mathrm{D}_{2} \mathrm{O}$ is expected at higher temperatures, ${ }^{2}$ which is consistent with the proposed multistep chain collapse during the coil-to-globule transiton, ${ }^{27,33}$ whereas for DP $=47$ and 40 , the resulted dimension of the polymers in $\mathrm{D}_{2} \mathrm{O}$ is comparable to or larger than that in toluene and methanol, which is due to finite interactions in the solutions and possible aggregation of a fraction of polymers in $\mathrm{D}_{2} \mathrm{O}$ due to strong end-group attractions. ${ }^{27}$ As will be demonstrated below, the end-group interactions facilitate the formation of the polymer dimers in aqueous solutions of short polymer chains with DP $=8$. We note that there are some differences between the polydispersity of the cross-section radius of the polymers in toluene, methanol, and in $\mathrm{D}_{2} \mathrm{O}$, which can be explained as being due to the relatively sharp interface between the polymer and the solvent in $\mathrm{D}_{2} \mathrm{O}^{27}$ as opposed to a rather diffuse interface in toluene and methanol solutions.

The data of the polymer with DP $=8$ were fitted to a phenomenological equation combining a form factor characterizing individual polymers and a term describing the scattering from clusters ${ }^{34}$ 


$$
I(q)=\frac{\varphi(\Delta \rho)^{2}}{V_{\mathrm{p}}} V_{\mathrm{p}}^{2} P(q)+\frac{S(0)}{\left\{1+[(D+1) / 3] \xi^{2} q^{2}\right\}^{D / 2}}
$$

Here $\xi$ is the characteristic size of the cluster, $D$ is the fractal dimension of the cluster, and $S(0)$ is proportional to the susceptibility of the system.

It was found that an ellipsoid form factor (eq A2 in the Appendix) best described the scattering curves from toluene and methanol solutions and a rigid cylinder form factor agreed with the data from the polymer solutions in $\mathrm{D}_{2} \mathrm{O}$. The reduced $\chi^{2}$ of the fitting was about 2 . The mass fractal dimension of the clusters is around 2. The obtained dimension of one ellipsoid in toluene and methanol is comparable to that of an individual polymer, the contour length of the PS backbone of which is estimated to be $8 \times 2.5=20 \AA$. The obtained contour length of the polymers includes both the length of the PS backbone and the contributions of the EG side chains near the chain end of the backbone. ${ }^{10 \mathrm{~d}}$ The length of the cylinder in $\mathrm{D}_{2} \mathrm{O}(L=65$ $\AA$, Table 1) is about 2 times that of one polymer chain if one takes into account the contributions from the side chains near the end group. We speculate that this dimension corresponds to the length of smaller aggregates, which consist of about two polymers linearly connected because of end-to-end interactions, as suggested by the computer simulation. ${ }^{27}$

\section{Summary}

SANS data demonstrate that the DP of the backbone is a key factor in controlling the structure of amphiphilic comblike PTrEGS polymers in various solvents. With the increasing DP of the backbone, the initially rigid polymers start to behave as flexible chains, the persistence length of which is mainly determined by the solvent-mediated interactions between EG side chains. The influence of the end groups on polymer-polymer interactions becomes especially pronounced at small DPs, that is, high relative concentration of end groups, especially in poor solvents like $\mathrm{D}_{2} \mathrm{O}$. As a result, clusterization gradually progresses in the $\mathrm{D}_{2} \mathrm{O}$ solutions of PTrEGS polymers with decreasing DP. Strong clustering is observed in all three solvents for polymers with a DP of 8 , and the sizes of the clusters are between 100 and $200 \AA$. The hydrophobic PS backbone of the polymers (e.g., $\mathrm{DP}=85$ and 58) is in a partially collapsed state in $\mathrm{D}_{2} \mathrm{O}$, whereas it expands in toluene and methanol. SANS data also suggest that the individual polymers assume the shape of a rigid cylinder with a relatively sharp interface in $\mathrm{D}_{2} \mathrm{O}$ and with a rather diffuse interface in toluene and methanol.

Acknowledgment. G.C. thanks Dr. Boualem Hammouda (NIST) for helpful discussions throughout the experiment at NIST. We thank Dr. Melissa Sharp and Dr. Vasyl Haramus (GKSS) for the help with SANS experiments. Research sponsored by the Division of Materials Sciences and Engineering, Office of Basic Energy Sciences, U.S. Department of Energy, under contract DEAC05-00OR22725 with Oak Ridge National Laboratory, managed and operated by UT-Battelle, LLC. Part of this research was conducted at the Center for Nanophase Materials Sciences, which is sponsored at ORNL by the Division of Scientific User Facilities, U.S. Department of Energy. G.C. and F.H. were partially supported by an appointment to the ORNL Postdoctoral Research Associates Program, administered jointly by the ORNL and the Oak Ridge Associated Universities. This work is based on activities supported in part by the National Science Foundation under agreement no. DMR-0454672 to the NIST Center for Neutron Research.

\section{Appendix}

1) The form factor of a rigid cylinder with a cross-section radius $R$ and length $2 H$ is given as ${ }^{23}$

$$
P(q)=\int_{0}^{\pi / 2}\left[2 j_{0}(q H \cos \alpha) \frac{J_{1}(q R \sin \alpha)}{q R \sin \alpha}\right]^{2} \sin \alpha \mathrm{d} \alpha
$$

where $V_{\text {cyl }}=\pi R^{2} L, H=L / 2, j_{0}(x)=\sin x / x$, and $J_{1}$ is the firstorder Bessel function.

2) The form factor of an ellipsoid with axes $r_{\mathrm{a}}$ and $r_{\mathrm{b}}$ is given $\operatorname{as}^{23}$

$$
P(q)=\frac{\phi}{V_{\text {ell }}}\left(\rho_{\text {ell }}-\rho_{\text {solv }}\right)^{2} \int_{0}^{1} F^{2}\left[q r_{\mathrm{b}}\left(1+x^{2}\left(v^{2}-1\right)\right)^{1 / 2}\right] \mathrm{d} x
$$

where

$$
F(z)=3 V_{\mathrm{ell}} \frac{\sin z-z \cos z}{z^{3}}, \quad V_{\mathrm{ell}}=\frac{4 \pi}{3} r_{\mathrm{a}} r_{\mathrm{b}}^{2}, \quad v=\frac{r_{\mathrm{a}}}{r_{\mathrm{b}}}
$$

3) The form factor of a semiflexible cylinder with the contour length $L \leq 4 b$, where $b$ is the Kuhn length, and a cross-section radius $R$ is given as follows ${ }^{32}$

$$
P(q, L, b)=P_{\text {chain }}(q, L, b)\left[2 J_{1}(q R) /(q R)\right]^{2}
$$

For $q b \leq q_{0}(L, b)$, where $q_{0}=\max \left\{1.9 /\left(<R_{g}{ }^{2}>1 / 2\right), 3\right\}$

$$
P_{\text {chain }}(q, L, b)=2[\exp (-u)+u-1] / u^{2}
$$

where

$$
\begin{gathered}
u=R_{\mathrm{g}}^{2} q^{2}, \quad R_{\mathrm{g}}=\left\langle R_{\mathrm{g}}^{2}\right\rangle^{1 / 2}, \quad\left\langle R_{\mathrm{g}}^{2}\right\rangle=\alpha(L / b)^{2}\left\langle R_{\mathrm{g}}^{2}\right\rangle_{0}, \\
\alpha(x)^{2}=\left[1+(x / 3.12)^{2}+(x / 8.67)^{3}\right]^{0.170 / 3}, \\
\left\langle R_{\mathrm{g}}^{2}\right\rangle_{0}=\frac{L b}{6}\left[1-\frac{3}{2 n_{\mathrm{b}}}+\frac{3}{2 n_{\mathrm{b}}^{2}}-\frac{3}{4 n_{\mathrm{b}}^{3}}\left[1-\exp \left(-2 n_{\mathrm{b}}\right)\right]\right], \quad n_{\mathrm{b}}=L / b
\end{gathered}
$$

For $q b>q_{0}(L, b)$

$$
P_{\text {chain }}(q, L, b)=\frac{a_{1}}{(q b)^{p_{1}}}+\frac{a_{2}}{(q b)^{p_{2}}}+\frac{\pi}{q L}
$$

where $p_{1}$ and $p_{2}$ are empirical constants and $p_{1}=5.36$ and $p_{2}=$ 5.62. $a_{1}$ and $a_{2}$ are given in ref 32 .

\section{References and Notes}

(1) Bolisetty, S.; Airaud, C.; Xu, Y.; Müller, A. H. E.; Harnau, L.; Rosenfeldt, S.; Lindner, P.; Ballauff, M. Phys. Rev. E 2007, 75, 040803 .

(2) Cheng, G.; Melnichenko, Y. B.; Wignall, G. D.; Hua, F.; Hong, K.; Mays, J. W. Polymer 2007, 48, 4108.

(3) Rathgeber, S.; Pakula, T.; Wilk, A.; Matyjaszewski, K.; Lee, H.-I.; Beers, K. L. Polymer 2007, 47, 7318

(4) Desvergne, S.; Héroguez, V.; Gnanou, Y.; Borsali, R. Macromolecules 2005, 38, 2400.

(5) Zhang, B.; Gröhn, F.; Pedersen, J. S.; Fisher, K.; Schmidt, M. Macromolecules 2006, 39, 8440.

(6) Feuz, L.; Leermakers, F. A. M.; Textor, M.; Borisov, O. Macromolecules 2005, 38, 8891 .

(7) Sheng, Y.-J.; Cheng, K.-L.; Ho, C.-C. J. Chem. Phys. 2004, 121, 1962.

(8) Elli, S.; Ganazzoli, F.; Timoshenko, E. G.; Kuznetsov, Y. A.; Connolly, R. J. Chem. Phys. 2004, 120, 6257.

(9) Duplessix, R.; Heroguez, V. Eur. Phys. J. E 2004, 15, 27.

(10) (a) Ito, K.; Kawaguchi, S. Adv. Polym. Sci. 1999, 142, 130. (b) Kawaguchi, S.; Akaike, K.; Zhang, Z. M.; Matsumoto, H.; Ito, K. Polym. J. 1998, 30, 1004. (c) Wataoka, I.; Urakawa, H.; Kajiwara, K.; Schmidt, M.; Wintermantel, M. Polym. Int. 1997, 44, 365. (d) Kikuchi, M.; Lien, N. T. L.; Narumi, A.; Jinbo, Y.; Izumi, Y.; Nagai, K.; Kawaguchi, S. Macromolecules 2008, 41, 6564.

(11) Liu, G.; Reinhout, M.; Mainguy, B.; Baker, L. G. Macromolecules 2006, 39, 4726.

(12) Zhang, W.; Shiotsuki, M.; Masuda, T. Macromolecules 2007, 40, 1421.

(13) Lutz, J.-F.; Weichenhan, K.; Akdemir, Ö.; Hoth, A. Macromolecules 2007, 40, 2503.

(14) Jonas, A.-M.; Glinel, K.; Oren, R.; Nysten, B.; Huck, W. T. S. Macromolecules 2007, 40, 4403.

(15) Ishizone, T.; Seki, A.; Hagiwara, M.; Han, S.; Yokoyama, A.; Oyane, A.; Deffieux, A.; Carlotti, S. Macromolecules 2008, 41, 2963. 
(16) Kuhlman, W. A.; Olivetti, E. A.; Griffith, L. G.; Mayes, A. M Macromolecules 2006, 39, 5122.

(17) Ito, K.; Kawaguchi, S. Colloids Surf., A 1999, 153, 173.

(18) Virtanen, J.; Tenhu, H. Macromolecules 2000, 33, 5970.

(19) Wu, C.; Qiu, X. Phys. Rev. Lett. 1998, 80, 620.

(20) (a) Glinka, C. J.; Barker, J. G.; Hammouda, B.; Krueger, S.; Moyer, J. J.; Orts, W. J. J. Appl. Crystallogr. 1998, 31, 430. (b) Wignall, G. D.; Bates, F. S. J. Appl. Crystallogr. 1987, 20, 18.

(21) Wignall, G. D.; Melnichenko, Y. B. Rep. Prog. Phys. 2005, 68, 1761

(22) Sheu, E. Phys. Rev. A 1992, 45, 2428.

(23) Guinier, A.; Fournet, G. In Small-Angle Scattering of X-Rays; John Wiley and Sons: New York, 1955.

(24) Dormidontova, E. E. Macromolecules 2004, 37, 7747.

(25) Hammouda, B.; Ho, D. L.; Kline, S. Macromolecules 2004, 37, 6932

(26) (a) Devanand, K.; Selser, J. C. Macromolecules 1991, 24, 5943. (b) Woodley, D. M.; Dam, C.; Lam, H.; LeCave, M.; Devanand, K.; Selser, J. C. Macromolecules 1992, 25, 5283. (c) Rangelov, S.; Brown, W. Polymer 2000, 41, 4825. (d) Rangelov, S.; Trzebicka, B.; JamrozPiegza, M.; Dworak, A. J. Phys. Chem. B 2007, 111, 11127.

(27) Vasilevskaya, V. V.; Klochkov, A. A; Lazutin, A. A.; Khalatur, P. G.; Khokhlov, A. R. Macromolecules 2004, 37, 5444.
(28) Wu, C.; Li, W.; Zhu, X. X. Macromolecules 2004, 37, 4989.

(29) Tokuhara, W.; Osa, M.; Yoshizaki, T.; Yamakawa, H. Macromolecules 2003, 36, 5311.

(30) Sanchez, I.; Lohse, D. J. Macromolecules 1981, 14, 131.

(31) (a) Koberstein, J. T.; Picot, C.; Benoit, H. Polymer 1985, 26, 673. (b) Medjahdi, G.; Sarazin, D.; François, J. Macromolecules 1991, 24, 4138. (c) Xie, Y.; Ludwig, K. F.; Bansil, R.; Gallagher, P. D.; Cao, X.; Morales, G. Physica A 1996, 232, 94. (d) Phillies, G. D. Macromolecules 2001, 34, 8745.

(32) (a) Pedersen, J. S.; Schurtenberger, P. Macromolecules 1996, 29, 7602. (b) Chen, W.-R.; Butler, P. D.; Magid, L. J. Langmuir 2006, 22, 6539. The fitting routine is included in the Igor data analysis package provided by NCNR of NIST.

(33) (a) Wu, C.; Wang, X. Phys. Rev. Lett. 1998, 80, 4092. (b) Chu, B.; Ying, Q.; Grosberg, A. Y. Macromolecules 1995, 28, 180. (c) Dasmahapatra, A. K.; Kumaraswamy, G.; Nanavati, H. Macromolecules 2006, 39, 9621.

(34) Shibayama, M.; Tanaka, T.; Han, C. C. J. Chem. Phys. 1992, 97, 6829.

MA801370Q 